Disponível em

http://www.anpad.org.br/rac

RAC, Rio de Janeiro, v. 18, n. 4, art. 4, pp. 446-464, Jul./Ago. 2014

http://dx.doi.org/10.1590/1982-7849rac20141193

(oc) EY-NO

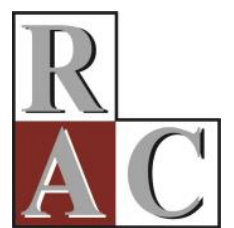

\title{
Níveis de Complexidade e Inserção em uma Rede Social de Comunidades Terapêuticas
}

\author{
Work Levels and Embeddedness in a Drug Rehab Center Social Network
}

Beatris Kemper Fernandes E-mail: beatriskf@yahoo.com.br

Universidade Positivo - UP

Rua Prof. Pedro Viriato Parigot de Souza, 5300, Campo Comprido, 81280-330, Curitiba, PR, Brasil.

Luis Gabriel Abravanel Santos

E-mail: tutorluis@hotmail.com Universidade Positivo - UP; Universidade Federal do Paraná - UFPR. Rua Prof. Pedro Viriato Parigot de Souza, 5300, Campo Comprido, 81280-330, Curitiba, PR, Brasil.

Bruno Henrique Rocha Fernandes E-mail: bruno@up.edu.br Universidade Positivo - UP Rua Prof. Pedro Viriato Parigot de Souza, 5300, Campo Comprido, 81280-330, Curitiba, PR, Brasil. 


\title{
Resumo
}

Vários são os estudos sobre intensidade das relações e força de atores em redes sociais dentro do âmbito organizacional, mas poucos incluem a ideia de níveis de complexidade - termo derivado do conceito de work levels. No presente estudo, conduziu-se uma pesquisa multinível numa rede composta por 38 comunidades terapêuticas (CT) de Curitiba-PR e Região Metropolitana. Investigou-se o nível de complexidade da atuação do dirigente e o posicionamento da CT numa rede intraorganizacional (quando filial de instituições multiunidades), dentro da rede interorganizacional e com instituições além desta rede. Graus de complexidade elevados se mostraram associados à centralidade interna (inDegree) na rede e à articulação com atores fora da rede; já o posicionamento na rede intraorganizacional não apresentou correlação com níveis de complexidade. Na rede estudada, indivíduos que trabalham em maior nível de complexidade tendem a ocupar uma posição mais central e realizam mais contatos fora da rede. A correlação entre níveis de complexidade do dirigente e centralidade na rede não significa necessariamente uma relação causal. Limita-se a afirmar que a relação existe e que, provavelmente, os dois movimentos ocorrem simultaneamente.

Palavras-chave: nível de complexidade; redes sociais; comunidades terapêuticas.

\begin{abstract}
There are several studies about the intensity of the relationships and strength of actors in a network within an organizational scope, but only a few include the concept of work levels. Thus, we conducted a multilevel survey in a network of 38 drug rehab centers (Comunidades Terapêticas [CT]) in the Region of Curitiba, Brazil. We investigated the $\mathrm{CT}$ leader's work level and the positioning of the CT in an intraorganizational net (when belonging to multi-branch institutions) within the network and with institutions beyond this network. High degrees of work levels were associated with internal network centrality (inDegree) and coordination with actors outside the network; intra-network positioning did not correlate with work levels. Higher work level leaders belonged to organizations with central positions in the network; they also manage more contacts outside the network. The correlation between the leaders' work levels and organization centrality in the network does not necessarily mean a causal relationship. We merely stated that the relationship exists and that probably the two movements occur simultaneously.
\end{abstract}

Key words: work levels; social networks; drug rehab centers. 


\section{Introdução}

O presente estudo visa compreender a relação entre capacidade de abstração de um ator e o posicionamento de sua organização em uma rede social. Para avaliar a capacidade de abstração, utilizase o conceito de níveis de complexidade, derivado da noção de work levels, proposto por Jaques (1978). Para analisar o posicionamento em uma rede, utiliza-se de conceitos e metodologias de análise de redes sociais, inicialmente, desenvolvidas, segundo Scott (2000), como forma de avaliar as estruturas sociais. Da década de 1930 aos anos de 1970, antropólogos, psicólogos e sociólogos desenvolveram o conceito de estrutura social (Freeman, 2006; Marineau, 1989; Mullins, 1973), culminando com as análises de redes sociais (Nohria \& Eccles, 1992). Tais estudos apontaram como as ações sociais são entrelaçadas, dando origem à imagem de rede social e às pesquisas sobre os elementos constitutivos dessas redes.

Para Powell e Smith-Doer (1994), as redes podem ser entendidas como forma de governança (abordagem relacional) ou ferramenta analítica (abordagem posicional). A abordagem relacional percebe as redes como forma de governança, visualizando-as como meios de acessar oportunidades, exercer poder e influenciar e estimular a inovação (Ibarra, 1993), meios de produção ou, ainda, instrumentos para compreender a organização como uma rede de tratados e acordos (Powell \& SmithDoer, 1994). A abordagem posicional enxerga as redes como ferramenta analítica e focaliza as relações sociais internas na organização, relações entre organizações ou das organizações com seu ambiente. Nos estudos organizacionais no Brasil, a análise de redes sociais tem sido utilizada por vários campos de conhecimento, como análise cientométrica (Rossoni \& Guarido, 2007), práticas organizacionais, respostas estratégicas, mudanças organizacionais (Bastos \& Santos, 2007), estruturas de governança (Lopes \& Baldi, 2009; Rossoni \& Machado-da-Silva, 2010), redes de desenvolvimento local (Casarotto \& Pires, 1999), relações de poder (Pinto \& Junqueira, 2008) e legitimidade (Rossoni \& Teixeira, 2008). Porém há uma carência de trabalhos que utilizem a metodologia de análise de redes associada ao conceito de níveis de complexidade ou work levels. Para Jaques (1978), work level refere-se à capacidade de abstração de um profissional para tomar decisões, considerando o número de variáveis envolvidas e suas implicações ao longo do tempo. Conforme será argumentado neste estudo, é de se esperar que profissionais com elevada capacidade de abstração, sobretudo dirigentes, atuem em organizações mais conectadas. No entanto uma pesquisa na base de dados EBSCO revelou poucos artigos que tangenciam a questão, como Hossain (2009), que constata que profissionais centralmente conectados numa rede coordenam melhor projetos; Tashiro, Lau, Mori, Fujii e Kajikawa (2012), que demonstraram a correlação entre centralidade numa rede de correio eletrônico e a performance de liderança dentro de uma organização; e Chung e Hossain (2009), que descobriram que a centralidade na rede influencia a performance de trabalhadores de conhecimento intensivo. Anteriormente, o trabalho de Ibarra (1993) apontou que indivíduos de maior centralidade numa rede estão mais propensos a inovar. Os estudos citados correlacionam a análise de redes a variáveis próximas ao conceito de níveis de complexidade (como coordenação em projetos, liderança em trabalhadores de conhecimento intensivo e capacidade de inovação), a existência de uma relação entre os níveis de complexidade de tarefa profissional e a centralidade que a organização possui em determinada rede social. Posto de outra forma, profissionais de organizações mais centrais em uma rede revelariam maior capacidade de abstração, leitura de cenários e atuação diante de um maior número de variáveis. Entretanto, se a relação é insinuada nos estudos, não é diretamente abordada, o que justifica a necessidade de mais pesquisas sobre a questão. Sobretudo, considerando a abordagem posicional (Powell \& Smith-Doer, 1994), pode-se verificar se relações sociais internas na organização, relações entre organizações ou no ambiente das organizações possuem igual relação sobre níveis de complexidade na atuação profissional.

A fim de estudar a relação entre níveis de complexidade e inserção numa rede, foi pesquisado o caso de uma rede de comunidades terapêuticas de Curitiba e Região Metropolitana (RMC), composta por 38 atores. Após a presente introdução, debate-se o quadro teórico de referência. A seguir, são apresentados e detalhados os procedimentos metodológicos adotados para a realização da pesquisa. Seguem-se os resultados e em seguida a análise de dados. Finalmente, são desenvolvidas as conclusões e considerações finais. 


\section{Quadro Teórico de Referência}

O termo rede foi utilizado em diversas perspectivas nos estudos organizacionais. Seu delineamento ocorreu a partir dos anos 30, do século XX, com estudos sobre estrutura social (Freeman, 2006). Na década de 1930, o psicólogo Jacob Levi Moreno realizou pesquisas sobre a interação social em pequenos grupos, utilizando a análise de redes para descrever, por meio da sociometria, as relações nos ambientes de trabalho e de estudos (Marineau, 1989). Na década de 1940, o sociólogo William Lloyd Warnere e o psicólogo Elton Mayo estudaram as relações interpessoais de exploração no ambiente de trabalho, na Universidade de Harvard (Mullins, 1973). Nesse período, o antropólogo RadcliffeBrown desenvolveu os conceitos de rede social. Nos anos de 1960 e 1970, estudos de Mark Granovetter, Harrison White e Barry Wellman popularizaram as análises de redes sociais (Nohria \& Eccles, 1992).

Segundo a definição de Manski (2000), redes sociais são estruturas compostas por indivíduos (ou organizações) chamados de nós, que estão conectados por um ou mais tipos de interdependência, como amizade, parentesco, interesses em comum, troca financeira, desagrado, relacionamentos, crenças, conhecimento ou prestígio. Ou seja, as redes englobam relacionamentos entre atores imersos em complexas e dinâmicas realidades sociais. Uma rede de relacionamentos consiste no somatório das interações entre membros de um determinado grupo, sem, necessariamente, levar em conta se é uma relação diádica (entre pares), um conjunto de membros ou um conjunto de ações (Whetten, 1981), nos quais transitam fluxos de informações, bens, serviços e influências (White, Boorman, \& Breiger, 1981). Nesta perspectiva, segundo Nohria e Eccles (1992), todas as organizações são redes sociais compostas por atores ligados por meio de relações sociais específicas. Assim, as redes são construídas socialmente, sendo reproduzidas e modificadas ao longo do tempo, como resultado da interação dos atores, cujas ações são influenciadas pela estrutura social.

Emirbayer e Goodwin (1994) apresentam duas formas de representar a estrutura social por meio da análise de redes: a abordagem relacional e a análise posicional. A abordagem relacional considera conexões diretas e indiretas entre os atores na rede. A análise posicional, com origem na sociologia e na teoria das organizações, concentra-se na posição ou papel que o conjunto de atores ocupa dentro de um sistema. Na abordagem posicional, as redes são percebidas como ferramenta analítica. Essa abordagem focaliza as relações sociais internas na organização (redes intraorganizacionais), relações entre organizações (redes interorganizacionais) ou no ambiente das organizações (relações entre redes).

Segundo Bastos e Santos (2007), o estudo das redes intraorganizacionais resulta da aplicação das ideias de redes sociais ao estudo de relações sociais internas da organização. Essa proposição parte do princípio de que uma organização pode ser vista internamente como uma rede de pessoas, de departamentos ou de setores específicos. A organização mantém uma constante rede de relações e significados compartilhados, caracterizada por uma subdivisão hierárquica, divisão de papéis e de atribuições de seus componentes. As redes interorganizacionais são constituídas de atores organizacionais que se relacionam por meio da troca de bens e serviços, influência e informações. Como em qualquer rede, seus integrantes possuem papel de maior ou menor centralidade, poder e influência.

As redes também podem ser consideradas para explicar a interação entre organizações, ou redes interorganizacionais (Powell \& Smith-Doer, 1994). Neste caso, a unidade de análise são as organizações integrantes da rede, sua interação se dá por meio de relações comerciais, parcerias para desenvolvimento de novos produtos ou atuação em negócios. Um recorte típico de tais redes consiste na indústria ou setor, com sua rede de fornecedores e clientes.

As redes interorganizacionais encontram-se posicionadas também em relação ao seu ambiente (Powell \& Smith-Doer, 1994). Sob este enfoque, pode-se analisar como os integrantes da rede interagem com elementos de outras redes, considerando a frequência e intensidade das interações, entre outros fatores. Powell e Smith-Doer (1994) exemplificam esse processo demonstrando como o conhecimento e a inovação difundem-se de uma determinada rede interorganizacional para outras como, por exemplo, a colaboração entre indivíduos da área de biotecnologia. 
Numa análise de redes, os nós ou pontos de ligação representam, por exemplo, pessoas ou organizações, e as linhas que ligam tais pontos representam um tipo de relacionamento. Os nós de uma rede são os atores que a compõem. A representação gráfica dessa rede é denominada sociograma (Nohria \& Eccles, 1992). O tamanho da rede é o total de ligações possíveis em uma matriz, descontando a diagonal, uma vez que um ator não pode se relacionar com ele mesmo. Já a densidade da rede é dada pelo quociente das ligações efetivamente existentes entre os atores da rede pelo total de ligações possíveis entre eles, dada pela equação $\mathrm{n} *(\mathrm{n}-1)$, sendo $\mathrm{n}$ é o valor de atores que compõem a rede (Borgatti, Everett, \& Freeman, 2012). Em uma rede é possível visualizar três medidas de centralidade, segundo Scott (2000): degree (centralidade de grau), closeness (centralidade de proximidade) ou betweenness (centralidade de intermediação). Degree diz respeito ao volume de contatos diretos, independentemente da qualidade desses contatos. A centralidade de grau de um ator significa "a identificação da posição em que se encontra em relação às trocas e à comunicação na rede" (Marteleto, 2001 , p. 76). Refere-se ao fato de o ator possuir contato ou não com outro ator e corresponde à quantidade de relações que se coloca entre um ator e outros atores. Closeness é relativo ao acesso e facilidade de relação, considerando a posição estrutural na rede. Seu cálculo é feito pelo levantamento do número de ligações que um ator deve percorrer para entrar em contato com qualquer outro membro da rede. Leva em conta a distância geodésica mais curta entre um ator e outro (farness, como denominado por Scott, 2000). A centralidade de proximidade é usada para identificar a distância entre centro e periferia na rede, sendo que o ator é "tão mais central quanto menor o caminho que ele precisa percorrer para alcançar os outros elos da rede" (Marteleto, 2001, p. 78). Finalmente, betweeness é relativo à posição de mediação de determinado nó com outros elos da rede. Utiliza-se para identificar contatos intermediários, que servirão como gatekeepers, ou pontes na rede. Assim, a centralidade de intermediação permite destacar os atores que possuem papel de corretores de conteúdo organizacional dentro de uma rede. Para Marteleto (2001, p. 79), a centralidade de intermediação mostra quanto um ator atua como "ponte", facilitando o fluxo de informação em uma determinada rede.

Em redes não simétricas, as medidas de centralidade podem ser relativas à entrada ou à saída. Medidas de entrada se referem a contatos recebidos de outros atores, já as de saída são de contatos à procura de outros atores (Scott, 2000). Por exemplo, uma medida de centralidade de grau, ou degree, terá dois valores: o inDegree, que corresponde à centralidade de grau de entrada e o outDegree, de saída. Segundo Freeman (1979), inDegree é a contagem do número de laços dirigidos para o ator e outDegree é o número dos laços dirigidos aos demais. Quando as relações estão associadas a aspectos positivos, como colaboração ou amizade, inDegree é, muitas vezes, interpretado como uma forma de popularidade e outDegree como uma medida mais gregária. A medida de centralidade está baseada no seguinte conceito: os vértices que possuem grau maior podem estar em uma posição mais privilegiada da rede. As medidas de centralidade de grau, ou degree, de um vértice $V$ para um determinado grafo $G:=(V, E)$ com $|V|$ vértices e $|E|$ arestas, é definido como $C_{D(v)}=\operatorname{deg}(v)$. É possível calcular a centralidade de grau para todos os nós em grafo $\Theta\left(V^{2}\right)$ em representação da densa matriz de adjacência do grafo, e para as arestas possui $\Theta(E)$ em representação de uma matriz dispersa. A definição da centralidade ao nível do nó pode ser estendida para o grafo inteiro. Sendo $v *$ o nó com o maior degree em $G, X:=(Y, Z)$ e Y conectado ao grafo, vale a seguinte equação (sendo Y o nó com maior grau de centralidade $\mathrm{X}$ ): $H=$ $\sum_{j=1}^{|Y|} C_{D}(Y *)-C_{D}(Y j)$ (Koschützki et al., 2005). O grau de centralidade do grafo $\mathrm{G}$ é dado por $C_{D}(G)=\frac{\sum_{i=1}^{|V|} C D(V *)-C_{D}(V i)}{H}$, sendo que o valor de $\mathrm{H}$ é maximizado quando o grafo $\mathrm{X}$ possui um nó central ao qual todos os demais nós são ligados; nesse caso $H=(n-1)(n-2)$. As medidas de centralidade de intermediação ou betweennes podem ser calculadas levando em conta $G$ como sendo um grafo conexo com $n$ vértices e sendo $v_{i}$ um vértice de $\mathrm{G}$. A medida de centralidade de intermediação de $v_{i}$, em percursos aleatórios, é dada por $C_{C A}\left(v_{i}\right)=\frac{2 \sum_{s<1} \tau_{s t}\left(v_{i}\right)}{n(n-1)}$. Ou seja, a centralidade de intermediação é definida a partir da média das distâncias geodésica do vértice $\mathrm{v}$ e todos os outros vértices alcançáveis por ele. Por fim, a centralidade de proximidade ou closeness é calculada por meio da fórmula $C_{C}(v)=$ $\sum_{t \in V / v} 2^{-d_{g G}}(v, t)$ (Koschützki et al., 2005). Esse tipo de centralidade considera o menor número de caminhos entre pares de vértices de uma rede, e os vértices que pertencem a um maior número de caminhos mínimos são os que possuem maior intermediação. 
Pode-se argumentar que atores de maior centralidade (seja de grau, proximidade ou intermediação) possuem maior capacidade de influência na rede, pois têm número maior de contatos (grau), facilidade de relação (proximidade) e poder de abrir portas (gatekeepers) para outros atores na rede (intermediação). Por extensão, também, podem ter maior visão sistêmica sobre a rede e seu contexto, visto que podem processar um maior volume de informações, o que lhes confere melhor visão do todo. Tais considerações remetem à noção de níveis de complexidade. O conceito de níveis de complexidade é derivado da ideia de work levels, proposta por Jaques (1978), ao sugerir que uma pessoa tende a executar seu trabalho dentro de um patamar de sofisticação, conforme suas atribuições e responsabilidades. Este nível tende a ser maior segundo o desenvolvimento e maturidade do profissional. Jaques (1978) o define em termos do número de variáveis com as quais uma pessoa tem de lidar dentro de um determinado tempo, numa dada situação, da clareza e precisão com a qual estas variáveis podem ser identificadas e sua velocidade de mudança. O work level está relacionado ao grau de abstração exigido para que o profissional tome uma decisão acertada e pode ser medido pelo tempo entre a tomada de decisão e as possibilidades de avaliação dos resultados desta decisão (chamado de time span). Profissionais que trabalham em maior nível de complexidade são capazes de articular um número maior de informações, antecipar tendências e acontecimentos, deduzir implicações e, diante disso, conseguem tomar, hoje, decisões acertadas cujo impacto se fará sentir anos adiante. Jaques (1978) sugere sete níveis de trabalho, ou work levels, cada um associado a intervalos de tempo que variam de três meses a mais de 20 anos, visão esta sustentada em trabalhos posteriores (Jaques, 1995; Jaques, Bygrave, \& Lee, 2001, Jaques \& Cason, 1994). Porém outros autores, como Rowbottom e Billis (1987), McMorland (2005) e Veloso e Dutra (2011), criticam a adoção exclusiva do parâmetro time-spam como único indicador do nível de complexidade com que alguém realiza seu trabalho. Tais autores propõem um conjunto maior de elementos, como: papel do gestor, resultados visados, contribuições almejadas para a organização, foco, tempo e objetivos. Sob essa ótica, o desenvolvimento profissional pode ser encarado como uma trajetória na qual o indivíduo vai se tornando capaz de trabalhar em matérias cada vez mais complexas, como ilustra a Figura 1.

\begin{tabular}{|c|c|c|c|c|c|c|}
\hline & Papel & Resultado & Contribuições & Foco & Tempo & Objetivos \\
\hline 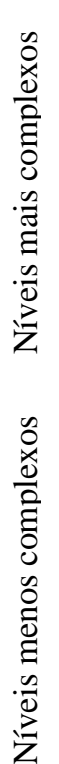 & $\begin{array}{c}\text { Presidente } \\
\text { internacional } \\
\text { Presidente de } \\
\text { grupo, Vice- } \\
\text { presidente } \\
\text { CEO/GM } \\
\text { Executivo } \\
\text { sênior } \\
\text { Gerente de } \\
\text { setor } \\
\text { Gestor de } \\
\text { primeira linha } \\
\text { Supervisor }\end{array}$ & $\begin{array}{c}\text { Prosperidade } \\
\text { Valor para os } \\
\text { Stakeholders } \\
\text { Retorno } \\
\text { sobre o } \\
\text { investimento } \\
\text { Mudança de } \\
\text { continuidade } \\
\text { Mudanças de } \\
\text { processos } \\
\text { Aumento de } \\
\text { eficiência } \\
\text { Melhora } \\
\text { contínua }\end{array}$ & $\begin{array}{c}\text { Adiciona } \\
\text { valores para } \\
\text { o futuro }\end{array}$ & $\begin{array}{c}\text { Desenvolvimento } \\
\text { Práticas } \\
\text { Serviços } \\
\text { Qualidade }\end{array}$ & $\begin{array}{c}\text { De } 20 \text { a } 50 \\
\text { anos } \\
\text { De } 10 \text { a } 20 \\
\text { anos } \\
\text { De } 5 \text { a } 10 \\
\text { anos } \\
\text { De } 2 \text { a } 5 \text { anos } \\
\text { De } 1 \text { a } 2 \text { anos } \\
\text { De } 3 \text { a } 12 \\
\text { meses } \\
\text { De } 0 \text { a } 3 \\
\text { meses }\end{array}$ & $\begin{array}{l}\text { Novas formas de } \\
\text { instituições } \\
\text { sociais, políticas e } \\
\text { econômicas } \\
\text { Visão, construção } \\
\text { de imagem } \\
\text { Direção } \\
\text { Inovação e } \\
\text { mudança } \\
\text { Práticas e sistemas } \\
\text { eficientes } \\
\text { Coordenação } \\
\text { eficiente } \\
\text { Excelência na } \\
\text { tarefa }\end{array}$ \\
\hline
\end{tabular}

Figura 1. Níveis de Complexidade das Atividades Organizacionais.

Fonte: Adaptado de McMorland, J. (2005). Are you big enough for your job? Is your job big enough for you? Exploring levels of work in organisations (p. 76). University of Auckland Business Review, 7(2), 75-83.

No presente artigo, procurou-se utilizar o termo níveis de complexidade para incluir as dimensões além do time span, segundo sugere o termo original work levels, de Jaques. Além disso, a tradução direta, níveis de trabalho, não refletiria o aumento da abstração e de complexidade que o presente estudo enfatiza. Estas razões justificam a opção pelo uso da expressão níveis de complexidade, 
encontrada nas obras no país (Dutra, 2010; Dutra, Hipólito, \& Silva, 2000; Fernandes, 2013; Veloso \& Dutra, 2011; Veloso, Silva, \& Dutra, 2012), para aproximar o conceito de work levels. O termo níveis de complexidade não está relacionado com a teoria da complexidade, defendida por autores como Edgar Morin (Morin, 1986).

No Brasil, a reflexão sobre níveis de complexidade/work levels vem recebendo atenção especial de autores ligados à área de gestão de pessoas, sobretudo gestão de competências (Dutra et al., 2000; Veloso et al., 2012). Nestes, tem sido constante a elaboração de matrizes para situar o nível de desenvolvimento profissional, tanto para efeitos de pesquisa como em projetos de intervenção (Fernandes, 2013; Veloso \& Dutra, 2011).

O conceito de níveis de complexidade aponta tanto para o desenvolvimento individual quanto para o organizacional. À medida que um indivíduo se desenvolve, tende a aumentar sua contribuição à organização, aplicando novos conhecimentos, implantando processos, ampliando e sofisticando tecnologias. Tal contribuição representa um legado ao patrimônio de conhecimentos da organização. A relação é recíproca: indivíduos buscam as organizações que percebem como referência em seu campo, pois sabem que a interação com o estado da arte potencializa seu desenvolvimento, permitindo-lhes alcançar atribuições de maior complexidade e abstração (Dutra, 2010; Veloso \& Dutra, 2011).

Internacionalmente, já existe alguma tradição em aplicar o conceito de work level (ou levels of work) a estudos empíricos. Por exemplo, Ivanov (2011) fez uma pesquisa longitudinal relacionando desempenho organizacional de empresas estatais norte-americanas com adequação entre organograma e work level da organização. Poucos trabalhos, porém, abordam a questão dos níveis de complexidade em conjunção com o conceito de redes. O trabalho sobre work levels de Clieaf (2004), por exemplo, não chega a abordar o tema de redes, mas, por força de suas constatações, sugere em sua conclusão a necessidade de se examinar os dois temas de forma combinada em pesquisas futuras. $\mathrm{O}$ autor constrói uma matriz de complexidade de cinco níveis para classificar work levels de chief executives officer (CEOs) de empresas de utilidade pública norte-americanas, relacionando-os ao grau de inovação destas empresas. Clieaf (2004) concluiu que praticamente metade dos CEOs concentra sua atenção em processos internos, em detrimento de uma visão estratégica voltada para fora da organização. Não atuam, portanto, em work levels de maior abstração, supostamente o demandado de um CEO. Tal constatação sugere a importância de se associar o conceito de work levels à análise de redes interorganizacionais, pois, segundo o autor, maior conexão externa do gestor sinaliza nível de complexidade mais amplo. Um dos poucos estudos que associam metodologia de redes sociais e work levels deve-se a Mattson (2012); porém o foco da autora foi investigar como altos níveis de complexidade no trabalho se relacionavam à qualidade de vida no trabalho de gestores.

O referencial teórico apresentado sugere, assim, haver espaço para abordar conjuntamente os temas níveis de complexidade e análise de redes, necessidade ainda mais premente face da escassez de estudos que examinem diretamente tal associação. A relação proposta é que há proporcionalidade direta entre o nível de interação da organização em uma rede social e o nível de complexidade de atuação de seus profissionais (Chung \& Hossain, 2009; Ibarra, 1993; Tashiro, Lau, Mori, Fujii, \& Kajikawa, 2012), particularmente, dos líderes organizacionais que, de forma geral, respondem pela formação e manutenção dos laços. Aplicando tal ideia à abordagem posicional da análise de redes (Powell \& SmithDoer, 1994), com redes intraorganizacionais, redes interorganizacionais e relações além da rede, três hipóteses podem ser formuladas:

H1: Líderes de organizações caracterizadas por uma intensa interação numa rede intraorganizacional tendem a atuar em níveis de complexidade elevados.

H2: Líderes de organizações caracterizadas por centralidade numa rede interorganizacional tendem a atuar em níveis de complexidade elevados.

H3: Líderes de organizações caracterizadas por intensa interação com organizações além da rede interorganizacional tendem a atuar em níveis de complexidade elevados. 
As três hipóteses destacam que a interação e exposição a situações diversas presentes em redes intra, inter e extraorganizacionais podem aumentar o repertório de experiências dos líderes organizacionais e, de modo inverso, líderes com elevada capacidade de abstração (nível de complexidade) podem mobilizá-las para construir e manter ligações. Desse modo, a lógica múltipla do estudo de redes, defendida por Powell e Smith-Doer (1994), embasa tais hipóteses.

\section{Procedimentos Metodológicos}

O presente estudo foi realizado em uma rede de comunidades terapêuticas atuante em Curitiba e RMC. Comunidades terapêuticas são organizações voltadas ao tratamento e recuperação de indivíduos dependentes químicos; devem ter licença das vigilâncias sanitárias dos estados ou municípios para funcionar, seguindo regulamentação da Agência Nacional de Vigilância Sanitária (ANVISA) (Resolução da Diretoria Colegiada [RDC] n. 101, 2001), e cadastro nos Conselhos Estaduais de Entorpecentes (CONEN) e Conselhos Municipais Antidrogas (COMAD/COMPED). O crescimento acelerado do tráfico e consumo de drogas e suas implicações colaboram com o agravamento da situação mundial relativa à saúde pública e à segurança. A Resolução RDC n. 101 (2001) estabeleceu normas mínimas relativas à estrutura física, recursos humanos necessários ao atendimento e metodologia de avaliação do tratamento, desde a triagem até a alta. Embora, em 2011, a RDC n. 101 tenha sido revogada e substituída pela RDC n. 29, na época da coleta de dados, ela ainda estava em vigor.

A rede de comunidades terapêuticas está organizada sob duas iniciativas: a Rede de Comunidades Terapêuticas de Curitiba e RMC (COMPACTA) e a Rede de Comunidades Terapêuticas da Secretaria Antidrogas Municipal. As comunidades terapêuticas integrantes da rede são convidadas a participar de reuniões, regularmente, e recebem habitualmente comunicados relevantes, como mudanças na legislação, editais para obtenção de recursos, convites para eventos, entre outros.

A rede foi escolhida por constituir um campo empírico propício à investigação das variáveis em questão, ou seja, permitiu capturar relacionamentos em redes intra e interorganizacionais e no ambiente, além de ser possível aferir o nível de complexidade de atuação do dirigente principal. Adicionalmente, cada comunidade da rede tem finalidades próximas (tratamento de dependentes químicos), são de pequeno porte e recursos limitados, o que as torna comparáveis e destaca o papel de sua liderança no sentido de diferenciar o escopo de atuação das organizações. Duas outras razões reforçam a escolha: o fato de tratar-se de um tipo alternativo de organização que foi poucas vezes abordado em estudos organizacionais - segundo Foucault (1984), organizações alternativas refletem melhor a sociedade como um todo, visto seguirem a lógica principal da sociedade civil $\left(3^{\circ}\right.$ setor $)$ e não tanto do estado $\left(1^{\circ}\right.$ setor $)$ e do mercado ( $2^{\circ}$ setor). Outra razão, de ordem prática, consiste em compreender estes aspectos para ajudar na melhoria da atuação destas organizações com função relevante na sociedade.

Todas as instituições pertencentes à rede foram pesquisadas. A relação das instituições foi obtida com a COMPACTA, totalizando-se 49 instituições de 12 municípios, são eles: Curitiba, Ponta Grossa, Lapa, Almirante Tamandaré, Araucária, Campina Grande do Sul, Campo Largo, Colombo, Mandirituba, Piraquara, Quitandinha e São José dos Pinhais. As instituições foram visitadas pessoalmente por um integrante da equipe de pesquisa a fim de realizar aplicação de um questionário adaptado da Secretaria Nacional de Políticas Sobre Drogas (SENAD), além de um questionário complementar com perguntas que visavam, entre outros objetivos, mapear a rede e o grau de conexão entre seus diversos membros. $\mathrm{O}$ questionário foi aplicado ao dirigente de cada instituição. O número de vagas para atendimento de dependente químicos dessas comunidades varia de 6 a 130, sendo que a média é de 37,39 vagas para pacientes, com desvio padrão de 27,89. Da amostra de 49 casos propostos inicialmente, dois foram descartados por não se configurarem como comunidade terapêutica: um era um grupo de teatro que atuava na recuperação de dependentes químicos e outro era um grupo de ajuda mútua. Nos casos em que o mesmo dirigente era responsável por mais de uma comunidade, apenas a instituição central foi contabilizada na análise. Nesse processo, o número de casos analisados se fixou em 38. A coleta de dados ocorreu no segundo semestre de 2009. Porém, a questão temporal não inviabiliza os achados deste 
artigo: o foco deste trabalho é investigar uma possível relação teórica entre work levels e atuação em rede - uma questão pouco estudada - e não descrever o estágio atual da rede analisada. Vale destacar que esta rede possui uma configuração relativamente estável, sendo que a imensa maioria das instituições que atuavam na época do levantamento ainda exerciam suas funções por ocasião da redação final deste trabalho.

O presente estudo é multinível, pois foca várias unidades de análise: (a) o gestor de determinada comunidade terapêutica; (b) a comunidade terapêutica, com interações com outras comunidades (rede interorganizacional), interações extra rede - com organizações além das terapêuticas - e, em alguns casos, a redes intraorganizacionais, quando pertencentes a instituições com várias unidades. Para Kozlowski e Klein (2000), há vários pressupostos teóricos que analisam a relação dos níveis macro (como organização ou grupo) com os níveis micro (por exemplo, indivíduos). Os estudos que se focam em fatores contextuais de níveis mais elevados que condicionam e influenciam os fenômenos de níveis inferiores (topo-base ou top-down) ou vice-versa (base topo ou bottom-up), podem ser considerados estudos multiníveis, como o presente estudo, que aborda o nível de análise individual (nível de complexidade) e as relações organizacionais em rede. Porém não é possível classificar tal estudo como topo-base ou base-topo, pois a relação entre tais elementos não se configura necessariamente em causalidade. Kozlowski e Klein (2000) ressaltam que essa classificação de estudo multinível aplica-se à teoria organizacional, que não deve ser confundida com modelos matemáticos desenvolvidos para analisar níveis diferentes de estudos entre a mesma população, como os modelos HLM, WABA, modelo de regressão pooled, Anova e Ancova.

Este estudo apresenta como variáveis o nível de complexidade do trabalho do dirigente da instituição, de um lado; e o nível de interação ou posicionamento da instituição intra, inter e além da rede de Comunidades Terapêuticas, de outro. A hipótese é que a centralidade na rede está relacionada ao nível de complexidade de atuação de seu dirigente. Mais especificamente, a hipótese da presente pesquisa é a de que a centralidade de grau de entrada (inDegree) dos atores da rede está relacionada com o nível de complexidade no qual tais atores atuam. Optou-se por analisar o conceito de centralidade por meio de medidas de entrada, o inDegree, que se refere à contagem do número de laços dirigidos para o ator, ou seja, o quanto os demais autores da rede o procuram. Assim, atores com maiores níveis de complexidade são mais procurados pelos demais e, de certa forma, os que contam com maior popularidade perante os demais. Com isso, são expostos a uma variedade maior de situações, o que aumenta seu estoque de experiências e conhecimentos. Esta acumulação de estoque lhes permite atuar como gatekeepers, o que lhes confere ascendência e reforça sua posição de centralidade.

Para aferir o nível de complexidade foi elaborada a matriz de complexidade da Tabela 1, fundamentada nas ideias de Jaques (1978), Rowbottom e Billis (1987), McMorland (2005), Clieaf (2004), Dutra (2010) e Fernandes (2013) e adaptada às especificidades do trabalho do dirigente de uma comunidade terapêutica. Foi elaborada pelos pesquisadores e validada, a seguir, por três especialistas da área: uma psicóloga, uma pedagoga e uma assistente social, com formação acadêmica e experiência de trabalho junto a tais instituições. Tal procedimento visou garantir validade de face e de conteúdo (Hair, Tatham, Anderson, \& Black, 2005). 
Tabela 1

\section{Matriz de Complexidade para Atuação da Comunidade}

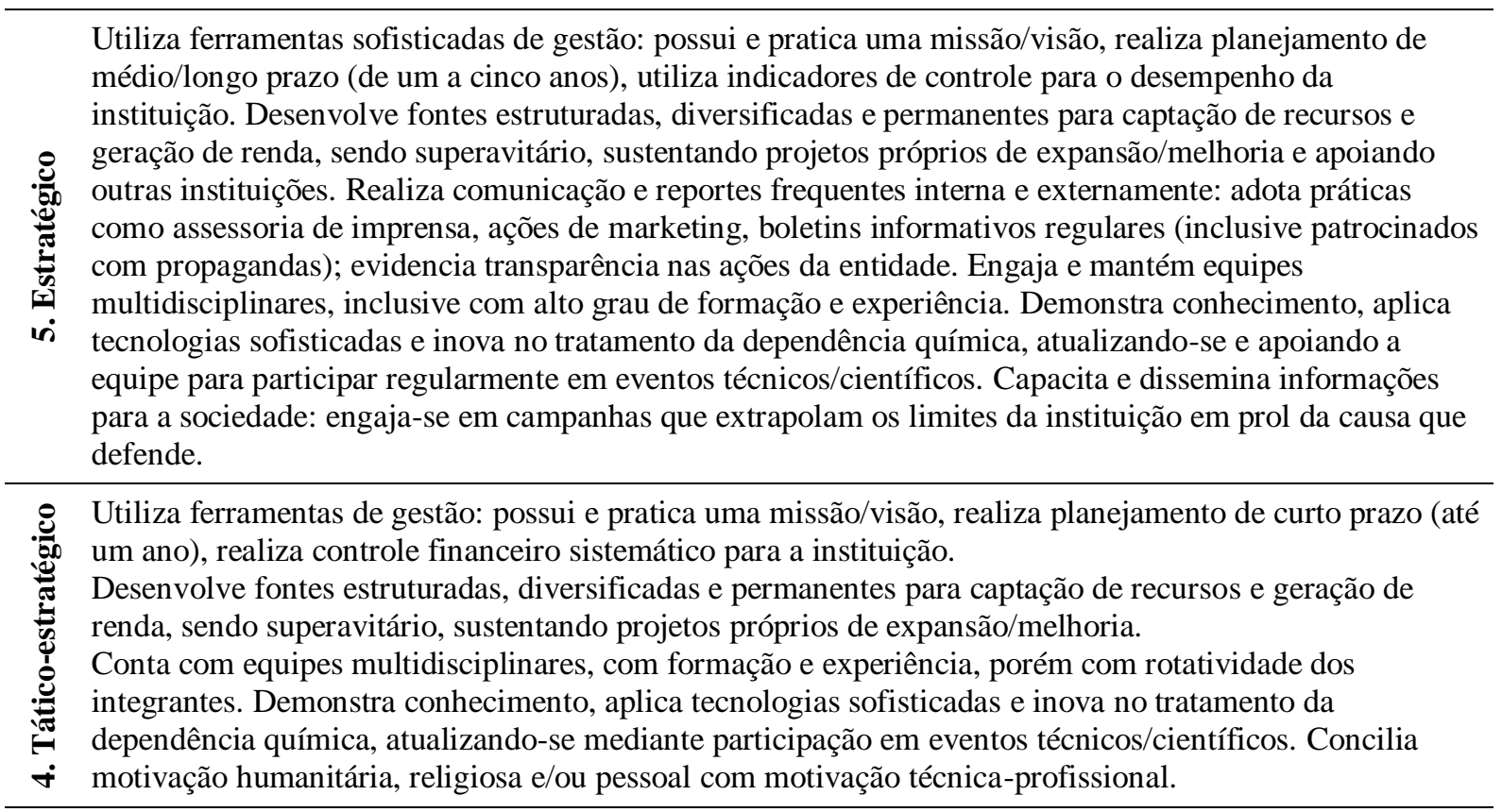

Demonstra conhecimento, aplica tecnologias tradicionais no tratamento da dependência química, atualizando-se esporadicamente em eventos técnicos/científicos. Desenvolve fontes estruturadas, diversificadas e permanentes para captação de recursos e geração de renda suficiente para o próprio sustento. Demonstra clareza dos objetivos institucionais, compartilhando-os com a equipe. Demonstra conhecimento, domínio e aplica integralmente regras da RDC 101.

\begin{tabular}{ll}
\hline Zela pela qualidade das instalações (infraestrutura, limpeza, etc.). Aplica metodologias reconhecidas e \\
tradicionais. Estabelece vínculos contratuais além dos voluntários. Demonstra conhecimento, mas \\
enfrenta dificuldade para aplicar integralmente regras da RDC 101 \\
\\
\hline
\end{tabular}

Nota. Fonte: Elaborada pelos autores.

Os dados obtidos nas entrevistas permitiram classificar os dirigentes em um dos níveis de complexidade propostos. O enquadramento se deu com base em elementos fáticos e evidenciáveis, calcados em atividades cotidianas dos dirigentes em suas instituições. Por exemplo, observou-se elementos como nível de formalização da entidade, uso de metodologias tradicionais ou próprias para o tratamento da dependência química, utilização de instrumentos de gestão, entre outros. Vale ressaltar que a matriz possui um caráter cumulativo, sendo que os níveis de maior abstração englobam os níveis de complexidade mais baixos. A outra variável refere-se ao grau de inserção ou centralidade na rede de comunidades terapêuticas. Esta rede foi dividida em três componentes. O primeiro refere-se à inserção em uma rede intraorganizacional (ou rede interna), aplicável no caso de instituições com mais de uma 
unidade sob a mesma direção - caso típico de ordens, congregações ou associações religiosas. Neste caso, procurou-se aferir se o pertencimento a uma associação representava algum estímulo para alavancar o nível de complexidade de atuação do dirigente. A hipótese seria de que sim, dado que experiências acumuladas no interior da associação poderiam ser transferidas a seus membros e dirigentes das unidades, propiciando seu desenvolvimento - articulando com o quadro teórico, o contato com o patrimônio de conhecimentos da instituição apoiaria o desenvolvimento do líder. Neste sentido, quanto maior a rede intraorganizacional, maiores as oportunidades de desenvolvimento. Esta variável foi categorizada em quatro grupos: (a) a CT não pertence a um grupo de organizações; (b) a CT pertence a um grupo de 1 a 5 organizações; (c) a CT pertence a um grupo de 6 a 10 organizações; (d) a CT pertence a um grupo com mais de 10 organizações. Outro componente referia-se à rede entre comunidades, rede interorganizacional ou simplesmente rede, que aponta para o relacionamento que as entidades têm umas com as outras. Aqui, observou-se, dentro da relação de 38 entidades, com quais organizações a comunidade terapêutica possuía relação. O dirigente poderia escolher quantas comunidades quisesse, entre as 38 listadas. O terceiro componente, as interações além-rede, focalizou os relacionamentos fora das comunidades terapêuticas, neste caso envolvendo governo, universidade, outras associações de natureza não terapêutica, entre outros. Foi identificado por meio da citação de entidades com as quais os dirigentes afirmaram manter relações. A variável foi contabilizada a partir do número de entidades externas relacionadas com cada comunidade terapêutica. O estudo trabalha em diferentes níveis de análise: individual, os dirigentes das comunidades, com foco no nível de complexidade; e organizacional, voltado a relacionamentos institucionais da entidade. Isso configura o trabalho como estudo multinível (Kozlowski \& Klein, 2000; Snijders \& Bosker, 2012).

\section{Apresentação dos Resultados}

A partir da matriz dos níveis de complexidade da Figura 2, os dirigentes foram classificados em um dos cinco níveis de complexidade, segundo suas atribuições cotidianas (Tabela 2). Das 38 comunidades, 12 foram classificadas em nível de abstração operacional, 10 em tático-operacional, 7 em tático, 4 em tático-estratégico e 5 em estratégico.

Tabela 2

Classificação das Comunidades em Níveis de Complexidade

\begin{tabular}{lllll}
\hline \multicolumn{1}{c}{$\mathbf{1}$ Operacional } & 2 Tático-operacional & \multicolumn{1}{c}{ 3 Tático } & 4 Tático-estratégico & 5 Estratégico \\
\hline N. Jerusalém & N. S. Gloria & Redenção & João Ceconello & IPTA \\
Betel & Só Vida & Reviver & CRENVI & ABAI \\
Hermon & Vida sem Drogas & CERENE & Monte Carmelo & CRAVI \\
CEJOCOMD & Desafio Jovem & Missão Shalom & Casa Bethânia & CTDia \\
Retorno ao Lar & CEIFAR & Casa Redentor & & 4 Pinheiros \\
Bibi Meirelles & Precavvida & Vida Nova & & \\
AMIVI & FAVI & Rosa Mística & & \\
Jesus é Rocha & FAZDI & & & \\
Toca de Assis & Belém & & & \\
ARCA & Emaús & & & \\
Mannain & & & & \\
Casa de Davi & & & & \\
\hline
\end{tabular}

Nota. Fonte: Dados primários. 


\section{Pertencimento à rede intraorganizacional}

Seguindo os procedimentos descritos, identificou-se se a comunidade terapêutica pertencia a alguma rede interna constituída por um grupo ou confederação. Os dados foram tabulados e classificados em quatro grupos. A Tabela 3 apresenta frequências e porcentagens. A única comunidade inserida em uma rede com mais de 10 participantes é a Toca de Assis.

Tabela 3

Tabela de Frequência de Pertencimento a uma Intrarrede

\begin{tabular}{lllll}
\hline & Número de comunidades em cada Grupo & Frequência & Porcentagem & Porcentagem acumulada \\
\hline Grupo 01 & 0 & 23 & 60,5 & 60,5 \\
Grupo 02 & 1 a 5 & 12 & 31,6 & 91,2 \\
Grupo 03 & 6 a 10 & 2 & 5,3 & 97,3 \\
Grupo 04 & Mais de 10 & 1 & 2,6 & 100 \\
\hline
\end{tabular}

Nota. Fonte: Dados primários.

\section{Rede interorganizacional das comunidades terapêuticas}

A rede analisada consistiu no conjunto de $38 \mathrm{CT}$. Para a análise desses dados foi utilizada a metodologia de análise de redes sociais. Primeiramente, os dados obtidos no questionário de redes foram tabulados em matrizes e, então, operacionalizados pelo software Ucinet versão 6.415, específico para a análise de redes e desenvolvido por Borgatti, Everett e Freeman (2012). Após lançados os dados no programa Ucinet, foram gerados matrizes, tabelas e sociogramas, fornecendo subsídios necessários para a planificação da estrutura de relacionamento da rede. A densidade da rede é de 0,1038 . Isso mostra que, de todas as 1.406 relações possíveis entre os 38 atores na rede, apenas 10,38\% se efetivam. O sociograma da rede de relacionamentos das comunidades terapêuticas está apresentado na Figura 2.

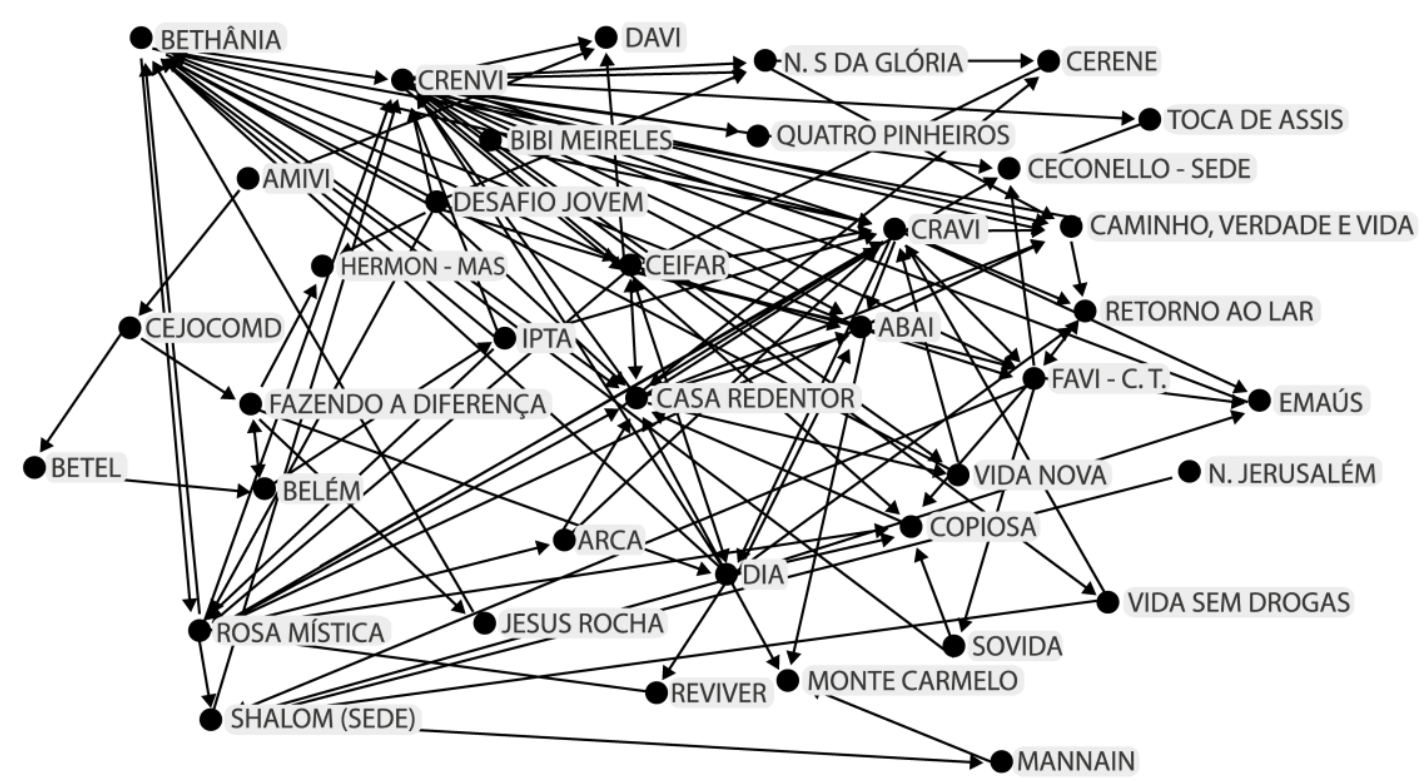

Figura 2. Sociograma da Rede de Relacionamentos das Comunidades de Curitiba e RMC. Fonte: Dados primários.

A partir da rede, foram identificadas algumas medidas de centralidade. A Tabela 4 ilustra as estatísticas descritivas das medidas de centralidade dos atores da rede analisada. Foram escolhidas 
medidas de centralidade de entrada, inDegree, inBetweenness e inCloseness, pois elas medem quanto o ator recebe de contatos da rede.

Tabela 4

Estatística Descritiva dos Dados de Centralidade da Rede Interorganizacional das Comunidades

\begin{tabular}{llcccc}
\hline & N & Mínimo & Máximo & Média & Desvio Padrão \\
\hline inDegree & 38 & 4167 & 58333 & 24768,56 & 3971,04 \\
inBetweenness & 38 & 1408 & 23515 & 5906,27 & 1280,41 \\
inCloseness & 38 & 32000 & 67606 & 50514,33 & 2270,99 \\
\hline
\end{tabular}

Nota. Fonte: Dados primários.

A correlação de Pearson entre centralidade de grau (inDegree) e centralidade de proximidade (inCloseness) foi alta $(0,917)$. Considera-se, então, que, nas comunidades terapêuticas estudadas, atores com maior número de relações também apresentam pequena distância para todos os atores da rede. Mesmo com menor força, a correlação entre centralidade de grau (inDegree) e centralidade de intermediação (inBetweenness) foi alta $(0,782)$, o que leva a entender que, na rede, comunidades com maior colaboração também são responsáveis por ligar comunidades isoladas a outros integrantes. Segundo Newman (2004), quando ocorre alta correlação entre indicadores de centralidade de grau e de intermediação, atores influentes se relacionam com atores influentes de outros grupos, o que pode demonstrar compartilhamento do conhecimento. A centralidade de proximidade e a de intermediação também apresentaram alta correlação $(0,738)$, o que, de certa forma, já era esperado, segundo o que se verificou em Newman (2004).

\section{Relações externas à rede de comunidades terapêuticas}

Os dados relativos às relações sistemáticas existentes com atores fora da rede foram tabulados e analisados com o SPSS 17. A estatística descritiva dos dados encontra-se na Tabela 5.

Tabela 5

Estatística Descritiva dos Dados das Relações Existentes com Atores Fora da Rede

\begin{tabular}{llcccc}
\hline & $\mathrm{N}$ & Mínimo & Máximo & Média & Desvio Padrão \\
\hline Articulação externa à rede & 38 & 0 & 12 & 4 & 0,48 \\
\hline
\end{tabular}

Nota. Fonte: Dados primários.

Nesse fator, a comunidade que mais possui relações fora da rede é a Quatro Pinheiros. Esses dados apenas contam o número de entidades parceiras citadas pelo gestor da comunidade na ocasião do levantamento, sem levar em conta a quantidade ou tipo de contatos. As entidades mais citadas foram a Secretaria Municipal de Assistência Social de Curitiba (SMAS), com 28 citações, a Secretaria Nacional de Políticas Sobre Drogas (SENAD) com 20 citações, o Conselho Tutelar e a Secretaria Municipal de Saúde de Curitiba, com 18 citações cada.

\section{Análise dos Resultados}

A partir do levantamento dos dados, as variáveis de relacionamento intraorganizacionais, interorganizacionais e além da rede de comunidades terapêuticas foram relacionadas com os dados 
obtidos pela matriz de nível de complexidade. Como a rede apresentou dados de centralidade de grau, proximidade e posição correlacionados, para evitar a multicolinearidade, foi selecionada apenas uma medida de centralidade para compor a análise de regressão. A medida escolhida foi a centralidade de grau (inDegree) porque, segundo Freeman (1979), é o conceito mais simples e puro de centralidade, sendo de ampla compreensão e aplicação em diversos estudos sociométricos. Além disso, Mizruchi e Marquis (2006) afirmam que variáveis de rede tendem a apresentar colinearidade, acarretando em sobreposição dessas variáveis. O inDegree também foi escolhido porque permite responder à hipótese do estudo, a saber, dirigentes com maiores níveis de complexidade estavam em organizações mais procuradas pelas demais. Porém a forte correlação entre a variável de centralidade de grau, de intermediação e de proximidade sugere que tais variáveis também são explicadas. Diante da hipótese delineada, na Tabela 6, estão expostos os resultados das regressões lineares OLS entre centralidade de grau em redes interorganizacionais, relações externas à rede de comunidades terapêuticas, pertencimento a uma rede intraorganizacional; e nível de complexidade. Análise de regressão é uma metodologia estatística que utiliza a relação entre duas ou mais variáveis de tal forma que uma variável pode ser predita a partir da outra ou de outras (Hair et al., 2005). Segundo Field (2009), há algumas condições que devem ser analisadas em um processo de regressão linear para avaliar a qualidade dos dados. Um dos procedimentos necessários é avaliar a normalidade das variáveis independentes e dependentes. Para isso, foi realizado o teste de Kolmogorov-Smirnov. Na variável inDegree, o teste se mostrou significativo $(\mathrm{P}<0,05)$ com o valor estatístico de 0,181 . Também com a variável de nível de complexidades, o teste se mostrou significativo com valor estatístico de 0,135. Desse modo, ambas as variáveis possuem distribuição normal. Outro procedimento é a avaliação dos resíduos das equações. Um dos problemas no processo de regressão é a correlação entre os erros das variáveis. Nesse sentido, é realizado o teste Durbin-Waston, que avalia a correlação com valores entre 0 e 4 . Valores próximos de 0 indicam uma correlação positiva, e, próximos de 4 , negativa. $O$ valor do teste na regressão entre as variáveis de nível de complexidade e inDegree foi de 1,333. Ou seja, há uma correlação positiva, mas para Field (2009), valores entre 1 e 3 são aceitáveis. A heterocedasticidade entre as variáveis foi avaliada por meio do gráfico de dispersão, que se mostrou como um histograma normal. Para a realização da regressão linear, Field (2009) recomenda o mínimo de 30 casos. No presente estudo são analisadas 38 instituições.

No Modelo 01, foi realizada regressão linear entre centralidade de grau na rede interorganizacional (inDegree), pertencimento a rede intraorganizacional e nível de complexidade. O Modelo 02 mostra uma regressão linear entre as articulações externas à rede de comunidades terapêuticas, pertencimento a uma rede intraorganizacional e nível de complexidade. Por fim, no Modelo 03, foi executada uma regressão linear múltipla pelo método Enter, levando em conta o inDegree, as articulações externas, pertencimento a uma rede intraorganizacional e níveis de complexidade. Pertencer a uma rede intraorganizacional não apresentou relação significativa em nenhum dos três modelos.

Tabela 6

Parâmetros Estimados para as Regressões Lineares

\begin{tabular}{lccc}
\hline \multirow{2}{*}{ Variáveis Independentes } & \multicolumn{3}{c}{ Nível de complexidade } \\
\cline { 2 - 4 } inDegree & Modelo 01 & Modelo 02 & Modelo 03 \\
Articulações externas & $0,590^{* * * *}$ & & $0,429^{* * *}$ \\
Pertencimento a um grupo de instituições & 0,021 & $0,623^{* * *}$ & $0,478^{* * *}$ \\
\hline $\mathrm{R}^{2}$ não ajustado & 0,349 & 0,111 & 0,047 \\
$\mathrm{R}^{2}$ ajustado & 0,331 & 0,388 & 0,551 \\
Significância & $<0,001$ & 0,371 & 0,525 \\
\hline
\end{tabular}

Nota. Fonte: Dados primários.

$* * *$ significante a $1 \%, * *$ significante a $5 \%, *$ significante a $10 \%$. 
Mediante a Tabela 6, pode-se visualizar que, no Modelo 01, a centralidade na rede, por meio do inDegree, explica 33,1\% da variância. No Modelo 02, as articulações externas têm um poder de explicação de 37,1\%. No Modelo 03, há um percentual de explicação maior, de 52,5\%. Os resultados trazem à tona conclusões interessantes. O nível de complexidade ou abstração do dirigente mostra-se correlacionado ao nível de articulação na rede. A centralidade (ou articulação), dentro da rede de comunidades, revelou-se significativa (Modelo 1); porém a articulação externa evidenciou maior associação com o nível de complexidade (Modelo 2). O Modelo 3 reforça tais achados ao atribuir maior coeficiente à rede externa do que à rede interorganizacional. $\mathrm{O}$ resultado atesta a associação entre nível de complexidade e grau de articulação em rede, apontando que a exposição a um maior repertório de experiências e contatos tende a enriquecer o nível de complexidade do dirigente e, de forma recíproca, o fato de trabalhar num nível de maior complexidade chancela uma posição de centralidade numa rede. Argumenta-se aqui a existência de uma relação recursiva, pois a metodologia utilizada não permite afirmar causalidade. Todavia há alguns elementos específicos da rede em análise que merecem ser comentados. O fato de a participação em redes externas ter maior associação com nível de abstração do que a inserção na rede intercomunidades sugere que o aprendizado ou experiências externas à rede podem ser elementos responsáveis por maior desenvolvimento do que a vivência exclusiva dentro da rede (ou, posto de outra forma, para não sugerir uma relação causal unidirecional, dirigentes com maior grau de complexidade estão expostos a mais vivências fora da rede de comunidades terapêuticas). $\mathrm{O}$ dado manifesta que o conhecimento de experiência além do mundo terapêutico, como interações com o governo, instituições educacionais, organismos internacionais, etc., pode ser fonte de aprendizado significativo para tais entidades, até maior do que o aprendizado extraído da convivência exclusiva dentro da rede. Tal experiência pode prover contato com mecanismos mais apurados de gestão (baseados em indicadores), prover acesso a metodologias de base mais científica e eficaz nos tratamentos, e mesmo mecanismos de financiamento sofisticados. O olhar apenas dentro da rede pode se revelar endógeno e, considerando o volume de instituições de atuação mais operacional, pode aportar pouco aprendizado, sobretudo aos dirigentes de entidades, que já operam em patamares mais elevados de complexidade. Caso sintomático desta relação é a CT Meninos de Quatro Pinheiros, que é a comunidade terapêutica que mais realiza articulações externas (12), inclusive com contatos internacionais, porém com centralidade de grau relativamente baixa (4167) dentro da rede. Olhando de perto o caso, trata-se de uma instituição de referência, com metodologias inovadoras em gestão e tratamento e boa parte desta abordagem provém de sua experiência externa à rede. Uma das explicações é que o alto nível de abstração pode fazer com que o ator privilegie contatos externos à rede, com outros atores com nível de complexidade semelhante ao dele. Isso reforça a ideia de que somente o estudo da rede isolada, sem levar em conta o meio externo, não explica totalmente os níveis de complexidade dos dirigentes da organização. Este caso revela também outra face: a baixa transferência deste aprendizado aos pares na rede, pelo menos de maneira sistemática e ampla, dado o baixo nível de relacionamento da entidade em questão. $\mathrm{O}$ fato de o componente intraorganizacional ter evidenciado baixa correlação com o nível de abstração dos dirigentes também é resultado curioso. Diferentemente do que se propôs como hipótese, observou-se que o pertencimento a uma rede intraorganizacional não provê aumento no nível de complexidade do dirigente ou, de forma inversa, dirigentes de instituições com várias unidades não necessariamente demonstram nível de abstração elevado. Isso mostra que nem sempre o pertencimento a um grupo ou rede, como a filiação a entidades mantenedoras, por exemplo, significa que a organização e seus indivíduos operem em um alto grau de complexidade. Assim, pode-se argumentar que estas organizações, ainda que tenham acesso a um repertório maior de experiências, não as promovem internamente para acelerar o desenvolvimento dos dirigentes de suas unidades. Uma segunda alternativa é que nem toda a experiência acumulada nestas instituições refira-se exclusivamente ao tratamento da dependência química - algumas destas organizações apresentam finalidade institucional mais ampla e apenas algumas de suas unidades destinam-se a dependentes químicos, sendo outras voltadas a focos diversos, como combate à pobreza ou reforço escolar. Desta forma, a transferência torna-se mais difícil, dada a distinção das áreas de atuação. Uma terceira possibilidade para o fato, considerando o assunto mais de perto, é que: muitas destas organizações são de natureza religiosa e confessional, com exigências de profissão de votos religiosos para assumir posições diretivas na organização. Diante da diminuição de vocações religiosas, tais instituições são forçadas a destinar a seus empreendimentos pessoas jovens e com pouca maturidade e experiência, tanto de gestão quanto nos temas técnicos relativos ao tratamento de dependência química. Diante disso, explica-se que não se encontre correlação entre grau de 
complexidade do dirigente e inserção em uma rede intraorganizacional. Por fim, analisando a parte de baixo das entidades, aquelas de baixa inserção na rede e baixo nível de complexidade na atuação do dirigente, observa-se uma postura muito mais voluntariosa do que técnica-profissional. Como padrão, trata-se de pessoas que tiveram alguma vivência relacionada ao consumo de drogas, tanto pessoal como na família e, mediante alguma experiência religiosa, sentem-se chamados a prestar um serviço em prol dos demais, pautados principalmente em suas crenças. Ou seja, procuram uma espécie de cura pela fé, motivo pelo qual também julgam, em boa parte, desnecessário buscar a aproximação e absorver experiências na rede, sejam internas às comunidades, sejam além das comunidades.

\section{Conclusões}

O presente estudo sugeriu ser possível integrar a metodologia e os conceitos de análise de redes sociais e o corpo teórico de níveis de complexidade. Isso mostra que, na rede analisada, indivíduos que trabalham em maior nível de complexidade atuam em organizações com uma posição mais central na rede e com mais contatos fora desta. A correlação entre níveis de complexidade do dirigente e centralidade na rede não significa necessariamente uma relação causal. Não é possível afirmar que indivíduos de maior grau de abstração levem suas entidades a relacionarem-se mais (e melhor); tampouco que o fato de haver um relacionamento mais qualificado promova o desenvolvimento do indivíduo. Um dos problemas e limitantes na utilização de regressões é que pesquisadores podem inferir uma relação de causa e efeito entre duas variáveis quando eles ajustam um modelo de regressão ou realizam uma análise de correlação. Uma associação significativa entre variáveis não necessariamente implica numa relação de causa e efeito. $O$ recorte da pesquisa não permite sustentar um movimento em detrimento de outro, mas se limita a afirmar que a relação existe e que, provavelmente, os dois movimentos aconteçam simultaneamente. E isso ocorre dentro da lógica proposta por Dutra (2010), ou seja, de que o indivíduo, ao se desenvolver, deixa seu legado à organização e vice-versa: a organização, ao disponibilizar aos indivíduos seu patrimônio de conhecimentos, de alguma forma, contribui para seu desenvolvimento. O pertencimento a uma rede intraorganizacional, por fim, não demonstrou associação com o nível de complexidade. Vários motivos podem estar por trás desta suposta ausência de transferência do legado organizacional ao indivíduo: a falta de mecanismos mais eficazes de gestão do conhecimento; a amplitude de áreas de atuação, algumas muito diversas, que dificultam a apropriação e adaptação dos conhecimentos de um campo em outro; ou a ausência de candidatos aptos para assumir funções diretivas. Tais suposições, porém, apenas foram lançadas e sua confirmação deve ser alvo de estudos posteriores. Também a ser melhor explorado é o achado referente ao voluntarismo das instituições de baixo nível de complexidade do dirigente e baixa articulação na rede. Supõe-se que a motivação religiosa ou pessoal assuma a tônica nestas entidades, a ponto de julgar que basta a fé como elemento para se cumprir a missão institucional e sua missão divina. Uma possível metodologia para continuar a investigação destas suposições são estudos de casos múltiplos a serem conduzidos nestas instituições. Tais estudos poderiam analisar e comparar as organizações de baixo nível de complexidade - baixa articulação em rede, aquelas de nível intermediário e as de alto nível de complexidade e atuação em rede, atentando à atuação do dirigente, a mecanismos de aprendizado e transferência de conhecimentos, entre outros. Na presente pesquisa, as instituições pertenciam a uma rede formal, a COMPACTA. Porém, consideradas as relações de fato, a rede informal mostrou-se pouco densa. Uma das possíveis explicações se dá pelo fato de a rede formal não estar plenamente institucionalizada, o que explica a existência de organizações com poucos laços. Este estudo não examinou se níveis de complexidade de inserção na rede associam-se a melhor performance organizacional. Tal questão também pode ser objeto de investigações futuras. Outra sugestão para pesquisas futuras é verificar a pertinência da relação entre níveis de complexidade e interação organizacional em outros contextos de redes sociais. 


\section{Referências}

Bastos, A. V., \& Santos, M. V. (2007). Redes sociais informais e compartilhamento de significados sobre mudança organizacional. Revista de Administração de Empresas, 47(3), 27-39. doi: $10.1590 / \mathrm{S} 0034-75902007000300003$

Borgatti, S., Everett, M., \& Freeman. L. (2012). UCINET 6.415 for Windows: software for social network analysis. Harvard, MA: Analytic Technologies.

Casarotto, N., Filho, \& Pires, L. H. (1999). Redes de pequenas e médias empresas e desenvolvimento local. São Paulo: Atlas.

Chung, K., \& Hossain, L. (2009). Measuring performance of knowledge-intensive workgroups through social networks. Project Management Journal, 40(2), 34-58. doi: 10.1002/pmj.20115

Clieaf, M. V. (2004). Are boards and CEOs accountable for the right level of work? Ivey Business Journal, 16(3), 1-12.

Dutra, J. S. (2010). Competências: conceitos e instrumentos para a gestão de pessoas na empresa moderna (2a ed.). São Paulo: Atlas.

Dutra, J. S., Hipólito, J. A. M., \& Silva, C. M. (2000). Gestão de pessoas por competências: o caso de uma empresa do setor de telecomunicações. Revista de Administração Contemporânea, 4(1), 161176. doi: 10.1590/S1415-65552000000100009

Emirbayer, M., \& Goodwin, J. (1994). Network analysis, culture and the problem of agency. American Journal of Sociology, 99(6), 1411-1454.

Fernandes, B. H. R. (2013). Gestão estratégica de pessoas com foco em competências. Rio de Janeiro: Elsevier.

Field, A. (2009). Discovering statistics using SPSS (3rd ed.). London: Sage.

Foucault, M. (1984). Microfísica do poder (4a ed., R. Machado, Trad. Org.). Rio de Janeiro: Edições Graal. (Obra original publicada em 1979)

Freeman, L. C. (1979). Centrality in social networks: conceptual clarification. Social Networks, 1(3), 215-239. doi: 10.1016/0378-8733(78)90021-7

Freeman, L. C. (2006). The development of social network analysis. Vancouver: Empirical Press.

Hair, J. F., Tatham, R. L., Anderson, R. E., \& Black, W. (2005). Análise multivariada de dados. Porto Alegre: Bookman.

Hossain, L. (2009). Effect of organizational position and network centrality on project coordination. International Journal of Project Management, 27(7), 680-689. doi: 10.1016/j.ijproman.2008.11.004

Ibarra, H. (1993). Network centrality, power, and innovation involvement: determinants of technical and administrative roles. Academy of Management Journal, 36(3), 471-501. doi: 10.2307/256589

Ivanov, S. (2011). Why organizations fail: a conversation about American competitiveness. The International Journal of Organizational Innovation, 4(1). 94-111.

Jaques, E. (1978). Levels of abstraction in logic and human action. London: Educational. 
Jaques, E. (1995). Why the psychoanalytical approach to understanding organizations is dysfunctional. Human Relations, 48(4), 343-350. doi: 10.1177/001872679504800401

Jaques, E., Bygrave, C., \& Lee, N. (2001). Aligning multiple time horizons and multiple functions in strategic planning and budgeting. International Journal of Organizational Analysis, 9(3), 257271. doi: $10.1108 / \mathrm{eb} 028935$

Jaques, E., \& Cason, K. (1994). Human capability a study of individual potential and its application. Virginia: Cason Hall \& Co. Publishers.

Koschützki, D., Lehmann, K. A., Peeters, L., Richter, S., Tenfelde-Podehl, D., \& Zlotowski, O. (2005). Centrality indices. In U. Brandes \& T. Erlebach (Eds.), Network analysis: methodological foundations (pp. 16-61). New York: Springer.

Kozlowski, S. W. J., \& Klein, K. J. (2000). Multilevel theory, research and methods in organizations: foundations, extensions, and new directions. San Francisco, CA: Jossey-Bass.

Lopes, F. D., \& Baldi, M. (2009). Redes como perspectiva de análise e como estrutura de governança: uma análise das diferentes contribuições. Revista de Administração Pública, 43(5), 1007-1035. doi: $10.1590 / \mathrm{S} 0034-76122009000500003$

Manski, C. F. (2000). Economic analysis of social interactions. Journal of Economic Perspectives, 14(3), 115-136. doi: 10.3386/w7580

Marineau, R. F. (1989). Ancestors and family: the birth of a myth. In R. F. Marineau (Ed.), Jacob Levy Moreno, 1889-1974: father of psychodrama, sociometry, and group psychotherapy (pp. 1-4), Londres: Routledge.

Marteleto, R. M. (2001). Análise de redes sociais: aplicação nos estudos de transferência da informação. Ciência da Informação, 30(1), 71-81. doi: 10.1590/S0100-19652001000100009

Mattson, C. (2012). Leadership stress in California community colleges (Tese de PhD). California State University, Fullerton, California.

McMorland, J. (2005). Are you big enough for your job? Is your job big enough for you? Exploring levels of work in organisations. University of Auckland Business Review, 7(2), 75-83.

Mizruchi, M. S., \& Marquis, C. (2006). Egocentric, sociocentric, or dyadic? Identifying the appropriate level of analysis in the study of organizational networks. Social Networks, 28(3), 187-208. doi: 10.1016/j.socnet.2005.06.002

Morin, E. (1986). Para sair do Século XX. Rio de Janeiro: Nova Fronteira.

Mullins, N. (1973). Theories and theory groups in contemporary American sociology. New York: Harper and Row.

Newman, M. E. J. (2004, January). Coauthorship networks and patterns of scientific collaboration. Proceedings of the National of Academic Sciences, Washington, DC, USA, 101.

Nohria, N., \& Eccles, R. G. (1992). Face-to-face: making network organizations work. In N. Nohria \& R. G. Eccles (Eds.), Networks and organizations: structure, form, and action (pp. 1-22). Boston, MA, USA: Harvard Business School Press.

Pinto, A. M. G., \& Junqueira, L. A. P. (2008). A análise de redes sociais como ferramenta de diagnóstico das relações de poder. eGesta, 4(1), 33-59. 
Powell, W. W., \& Smith-Doer, L. (1994). Networks and economic life. In N. I. Smelser \& R. Swedberg (Eds.), Handbook of economic sociology (pp. 368-402). Princeton, NJ, USA: Princeton Russell Sage Foundation.

Resolução da Diretoria Colegiada n. 101, de 30 de maio de 2001. (2001). Estabelece as diretrizes para a assistência extra-hospitalar aos portadores de transtornos mentais. Brasília, DF: ANVISA. Recuperado http://www.saude.rs.gov.br/upload/20120425144823rdc_n_101_02_ms__anvisa.pdf

Resolução da Diretoria Colegiada n. 29, de 30 de junho de 2011. (2011). Dispõe sobre os requisitos de segurança sanitária para o funcionamento de instituições que prestem serviços de atenção a pessoas com transtornos decorrentes do uso, abuso ou dependência de substâncias psicoativas. Brasília, DF: ANVISA. Recuperado de http://www.pactopoa.com.br/?p=180

Rossoni, L., \& Guarido, E., Filho (2007). Cooperação interinstitucional no campo da pesquisa em estratégia. Revista de Administração de Empresas, 47(4). 74-88. doi: 10.1590/S003475902007000400007

Rossoni, L., \& Machado-da-Silva, C. L. (2010). Institucionalismo organizacional e práticas de governança corporativa [Edição Especial]. Revista de Administração Contemporânea, 14, 173 198. doi: 10.1590/S1415-65552010000600008

Rossoni, L., \& Teixeira, R. M. A. (2008). Interação dos relacionamentos com os recursos e a legitimidade no processo de criação de uma organização social. Cadernos EBAPE.BR, 6(4), 1-19.

Rowbottom, R., \& Billis, D. (1987). Organizational design: the work-level approach. Aldershot: Gower Publishing.

Scott, J. (2000). Social network analysis (2nd ed.). Thousand Oaks, California: SAGE Publ.

Snijders, T. A. B., \& Bosker, R. J. (2012). Multilevel analysis: an introduction to basic and advanced multilevel modeling (2nd ed.). London: Sage Publishers.

Tashiro, H., Lau, A., Mori, J., Fujii, N., \& Kajikawa, Y. (2012). E-mail networks and leadership performance. Journal of the American Society for Information Science \& Technology, 63(3), 600606. doi: 10.1002/asi.21667

Veloso, E. F. R., \& Dutra, J. S. (2011). Carreiras sem fronteiras na gestão pessoal da transição profissional: um estudo com ex-funcionários de uma instituição privatizada. Revista de Administração Contemporânea, 15(5), 834-854. Recuperado de http://www.scielo.br/pdf/rac/v15n5/a04v15n5.pdf. doi: 10.1590/S1415-65552011000500004

Veloso, E. F. R., Silva, R. C., \& Dutra, J. S. (2012). Diferentes gerações e percepções sobre carreiras inteligentes e crescimento profissional nas organizações. Revista Brasileira de Orientação Profissional, 13(2), 197-207.

Whetten, D. A. (1981). Interorganizational relations: a review of the field. Journal of Higher Education, $52(1), 1-28$.

White, H. C., Boorman, S. A., \& Breiger, R. L. (1981). Social structure from multiple networks: role structures. American Journal of Sociology, 81(4), 384-446. 